\title{
Coherent configurations, association schemes and permutation groups
}

\author{
Peter J. Cameron \\ School of Mathematical Sciences \\ Queen Mary, University of London \\ London E1 4NS \\ U.K. \\ $p \cdot j \cdot c a m e r o n @ q m u l \cdot a c \cdot u k$
}

\begin{abstract}
Coherent configurations are combinatorial objects invented for the purpose of studying finite permutation groups; every permutation group which is not doubly transitive preserves a non-trivial coherent configuration. However, symmetric coherent configurations have a much longer history, having been used in statistics under the name of association schemes.

The relationship between permutation groups and association schemes is quite subtle; there are groups which preserve no non-trivial association scheme, and other groups for which there is not a unique minimal association scheme.

This paper gives a brief outline of the theory of coherent configurations and association schemes, and reports on some recent work on the connection between association schemes and permutation groups.
\end{abstract}

\section{Coherent configurations}

This section contains the definitions of coherent configurations and of various specialisations (including association schemes), and their connection with finite permutation groups. It is by no means a complete survey of this topic, but it includes some historical remarks. 


\subsection{Definitions}

Let $\Omega$ be a finite set. A coherent configuration on $\Omega$ is a set $\mathcal{P}=\left\{R_{1}, \ldots, R_{S}\right\}$ of binary relations on $\Omega$ (subsets of $\Omega^{2}$ ) satisfying the following four conditions:

(a) $\mathcal{P}$ is a partition of $\Omega^{2}$;

(b) there is a subset $\mathcal{P}_{0}$ of $\mathcal{P}$ which is a partition of the diagonal $\Delta=\{(\alpha, \alpha): \alpha \in$ $\Omega\}$;

(c) for every relation $R_{i} \in \mathcal{P}$, its converse $R_{i}^{\top}=\left\{(\beta, \alpha):(\alpha, \beta) \in R_{i}\right\}$ is in $\mathcal{P}$; say $R_{i}^{\top}=R_{i^{*}}$

(d) there exist integers $p_{i j}^{k}$, for $1 \leq i, j, k \leq s$, such that,for any $(\alpha, \beta) \in R_{k}$, the number of points $\gamma \in \Omega$ such that $(\alpha, \gamma) \in R_{i}$ and $(\gamma, \beta) \in R_{j}$ is equal to $p_{i j}^{k}$ (and, in particular, is independent of the choice of $\left.(\alpha, \beta) \in R_{k}\right)$.

The numbers $p_{i j}^{k}$ are called the intersection numbers of the coherent configuration $\mathcal{P}$. (They are so-called because $p_{i j}^{k}$ is the cardinality of the intersection $R_{i}(\alpha) \cap R_{j}^{\top}(\beta)$ for $(\alpha, \beta) \in R_{k}$, where $R(\alpha)=\{\beta \in \Omega:(\alpha, \beta) \in R\}$.)

We can represent a binary relation $R$ on $\Omega$ by its basis matrix $A(R)$, whose rows and columns are indexed by $\Omega$, and whose $(\alpha, \beta)$ entry is 1 if $(\alpha, \beta) \in R$, 0 otherwise. Using these matrices, and letting $I$ and $J$ be the identity and all-1 matrices, the axioms take the following form:

(a) $\sum_{i=1}^{S} A\left(R_{i}\right)=J$

(b) $\sum_{i=1}^{t} A\left(R_{i}\right)=I$, where $\left\{R_{1}, \ldots, R_{t}\right\}$ is the subset referred to in (b) above.

(c) For each $i$, there exists $i^{*}$ such that $A\left(R_{i}\right)^{\top}=A\left(R_{i^{*}}\right)$.

(d) For each pair $i, j$, we have

$$
A\left(R_{i}\right) A\left(R_{j}\right)=\sum_{k=1}^{s} p_{i j}^{k} A\left(R_{k}\right)
$$

It follows from (b) and (d) that the span of $\left\{A\left(R_{1}\right), \ldots, A\left(R_{S}\right)\right\}$ (over the complex numbers) is an algebra, and from (c) that this algebra is semisimple (and so is 
isomorphic to a direct sum of matrix algebras over $\mathbb{C}$ ). This algebra is called the basis algebra of the configuration. We denote the basis algebra of $\mathcal{P}$ by $\mathrm{BA}(\mathcal{P})$. Note that $\mathrm{BA}(\mathscr{P})$ consists of all the functions from $\Omega^{2}$ to $\mathbb{C}$ which are constant on the parts of $\mathcal{P}$.

Moreover, if $P_{j}$ is the $s \times s$ matrix with $(i, k)$ entry $p_{i j}^{k}$, then the map $A\left(R_{j}\right) \mapsto P_{j}$ for $j=1, \ldots, s$ extends linearly to an algebra isomorphism. (Indeed, Equation (1) shows that this map is the regular representation of $\mathrm{BA}(\mathcal{P})$, written with respect to the basis matrices.) Thus the matrices $P_{1}, \ldots, P_{S}$ also span an algebra, called the intersection algebra of $\mathcal{P}$.

The irreducible modules for the intersection algebra, and their multiplicities in the module $\mathbb{C} \Omega$, can be calculated from the intersection numbers. The multiplicities must of course be non-negative integers. This is one of the most powerful methods for showing nonexistence of coherent configurations with given intersection numbers. See Higman [16] for an early application to permutation groups.

Many familiar algebraic and combinatorial objects are coherent configurations. These include strongly regular and distance-regular graphs, symmetric and quasi-symmetric designs, partial geometries, generalised polygons, difference sets, and Schur rings. Moreover, as Delsarte [9] showed, the study of special subsets of coherent configurations provides a common context for much of design theory and coding theory. Delsarte also introduced new methods (such as linear programming) in this general context and applied them to both codes and designs.

Two extreme examples of coherent configurations will be important to us:

- The trivial configuration on $\Omega$ consists of the two relations $E$ and $\Omega^{2} \backslash E$, where $E=\{(\alpha, \alpha): \alpha \in \Omega\}$ is the diagonal (the relation of equality).

- The discrete configuration on $\Omega$ is the partition of $\Omega^{2}$ into singleton sets.

\subsection{Permutation groups}

If $G$ is any permutation group on $\Omega$, then the partition of $\Omega^{2}$ into orbits of $G$ is a coherent configuration, which we denote by $\mathcal{K}(G)$. We refer to this as the group case; a coherent configuration of the form $\mathcal{K}(G)$ is called Schurian. The trivial and discrete c.c.s are Schurian, corresponding to the symmetric group and the identity group respectively. Indeed, D. G. Higman [16, 17] introduced coherent configurations in order to study permutation groups, as the title of his early lecture notes [18] suggests.

An automorphism of a partition $\mathcal{P}$ of $\Omega^{2}$ is a permutation of $\Omega$ which fixes every set in $\mathcal{P}$; a weak automorphism is a permutation which maps each member 
of $\mathcal{P}$ to a member of $\mathcal{P}$. The automorphisms of $\mathcal{P}$ form a group, the automorphism group of $\mathcal{P}$, denoted by $\operatorname{Aut}(\mathcal{P})$. We will be concerned almost exclusively with automorphisms (which are sometimes called strict automorphisms); but we note the following fact. (The order relation on partitions is the usual one, which will be discussed further in the next section.)

Proposition 1.1 Let $G$ be a group of weak automorphisms of the coherent configuration $\mathcal{P}$ on $\Omega$. Let $\mathcal{P}^{G}$ be the partition of $\Omega$ whose parts are the unions of the G-orbits on the parts of $\mathcal{P}$. Then $\mathcal{P}^{G}$ is a coherent configuration; it is the unique finest coherent configuration coarser than $\mathcal{P}$ which admits $G$ as a group of automorphisms.

We note also that $G$ is a group of automorphisms of the coherent configuration $\mathcal{K}(G)$; that is, $G \leq \operatorname{Aut}(\mathcal{K}(G))$. The group $G$ is said to be 2-closed if $\operatorname{Aut}(\mathcal{K}(G))=G$ : that is, any permutation of $\Omega$ which fixes every $G$-orbit on pairs belongs to $G$. There is thus a bijection between Schurian coherent configurations and 2-closed permutation groups on $\Omega$.

\subsection{Some special coherent configurations}

Let $\mathcal{P}$ be a coherent configuration on $\Omega$. The sets $F$ such that $\{(\alpha, \alpha): \alpha \in F\}$ belong to $\mathcal{P}$ are called the fibres of $\mathcal{P}$; they form a partition of $\Omega$. We say that $\mathcal{P}$ is homogeneous if there is only one fibre. If $\mathcal{P}=\mathcal{K}(G)$, the fibres of $\mathcal{P}$ are the orbits of $G$ on $\Omega$; so $\mathcal{K}(G)$ is homogeneous if and only if $G$ is transitive.

Table 1 gives the numbers of homogeneous coherent configurations on small numbers $n$ of points ( $n \leq 30, n \neq 29$ ). These configurations have been computed by A. Hanaki and I. Miyamoto [15], and are available from Hanaki's Web page (which gives the configurations explicitly). The numbers up to 23 are cited by Bannai [5, p. 48]. By checking which of the configurations are Schurian, we obtain the numbers in the third column of the table. These numbers can also be calculated in another way. Alexander Hulpke [8, 20, 21] has computed the transitive permutation groups of degree at most 29. Those of degree at most 23 are included in GAP [13]; I am grateful to Alexander for providing me with data on larger degrees. We can check which of these are 2-closed, either by using a builtin GAP function, or more efficiently using nauty [28], which is interfaced with GAP using the share package GRAPE [32]. Reassuringly, all methods give the same answer! The values for $n=29$ follow from another result of Hanaki and Miyamoto (personal communication) that any non-Schurian homogeneous coherent configuration on 29 points is a complementary pair of strongly regular graphs, 
together with the result of Spence [33] that there are 41 strongly regular graphs on 29 points, of which only one is self-complementary. The last two columns of the table will be described later in this section.

The table shows that, on small numbers of points, most homogeneous coherent configurations arise from groups. (Indeed, the smallest non-Schurian example has 15 points; it is a "strongly regular tournament", whose automorphism group has order 21 and has three orbits on points.) This pattern is unlikely to hold in general; it is plausible that the proportion which are Schurian tends to zero as the number of points increases. Pyber [30] gives some results on the number of subgroups of $S_{n}$; it is likely that only a small proportion of these are 2-closed. However, only in special cases such as particular strongly regular graphs do good estimates for the numbers of coherent configurations exist.

The combinatorial explosion is not revealed by the data in Table 1, although it probably begins shortly after this point. For example, for $n=36$, McKay and Spence [29] have shown that there are 32548 strongly regular graphs with parameters $(36,15,6,6)$ (these are particular homogeneous coherent configurations), of which only one is Schurian!

A homogeneous c.c. is called thin if all basis matrices have row and column sums 1. A thin homogeneous c.c. is Schurian, and arises from a regular permutation group. For this reason, homogeneous c.c.s are sometimes called generalized groups [36].

A coherent configuration is called symmetric if all the relations are symmetric. A symmetric c.c. is homogeneous. (For, given any relation $R$ in a c.c. with fibres $F_{1}, \ldots, F_{t}$, there are indices $i, j$ such that $R \subseteq F_{i} \times F_{j}$.) If $\mathcal{P}=\mathcal{K}(G)$, then $\mathcal{P}$ is symmetric if and only if $G$ is generously transitive, that is, any two points of $\Omega$ are interchanged by some element of $G$.

Let $\mathcal{P}$ be a c.c. on $\Omega$. The symmetrisation $\mathcal{P}^{\text {sym }}$ of $\mathcal{P}$ is the partition of $\Omega^{2}$ whose parts are all unions of the parts of $\mathcal{P}$ and their converses. It may or may not be a c.c.; if it is, we say that $\mathcal{P}$ is stratifiable. The name, arising in statistics [2], will be explained later. It can be shown that, if $\mathcal{P}=\mathcal{K}(G)$, then $\mathcal{P}$ is stratifiable if and only if the permutation representation of $G$ is real-multiplicity-free, that is, if it is decomposed into irreducibles over $\mathbb{R}$, they are pairwise non-isomorphic. (Equivalently, the complex irreducibles have multiplicity at most one except for those of quaternionic type, that is, Frobenius-Schur index -1 , which may have multiplicity 2.)

Finally, a coherent configuration is called commutative if its basis matrices commute with one another. It can be shown that, if $\mathcal{P}=\mathcal{K}(G)$, then $\mathcal{P}$ is commutative if and only if the permutation representation is (complex)-multiplicity-free. 


\begin{tabular}{|c|c|c|c|c|}
\hline $\begin{array}{c}\text { Number of } \\
\text { points }\end{array}$ & $\begin{array}{l}\text { Homogeneous } \\
\text { c.c.s }\end{array}$ & $\begin{array}{l}\text { 2-closed } \\
\text { trans. groups }\end{array}$ & $\begin{array}{c}\text { Association } \\
\text { schemes }\end{array}$ & $\begin{array}{l}\text { 2-closed, gen. } \\
\text { trans. groups }\end{array}$ \\
\hline 1 & 1 & 1 & 1 & 1 \\
\hline 2 & 1 & 1 & 1 & 1 \\
\hline 3 & 2 & 2 & 1 & 1 \\
\hline 4 & 4 & 4 & 3 & 3 \\
\hline 5 & 3 & 3 & 2 & 2 \\
\hline 6 & 8 & 8 & 4 & 4 \\
\hline 7 & 4 & 4 & 2 & 2 \\
\hline 8 & 21 & 21 & 10 & 10 \\
\hline 9 & 12 & 12 & 6 & 6 \\
\hline 10 & 13 & 13 & 8 & 8 \\
\hline 11 & 4 & 4 & 2 & 2 \\
\hline 12 & 59 & 59 & 21 & 21 \\
\hline 13 & 6 & 6 & 4 & 4 \\
\hline 14 & 16 & 16 & 8 & 8 \\
\hline 15 & 25 & 24 & 10 & 10 \\
\hline 16 & 222 & 206 & 63 & 56 \\
\hline 17 & 5 & 5 & 4 & 4 \\
\hline 18 & 95 & 93 & 32 & 32 \\
\hline 19 & 7 & 6 & 3 & 3 \\
\hline 20 & 95 & 95 & 41 & 41 \\
\hline 21 & 32 & 32 & 11 & 11 \\
\hline 22 & 16 & 16 & 8 & 8 \\
\hline 23 & 22 & 4 & 2 & 2 \\
\hline 24 & 750 & 669 & 157 & 136 \\
\hline 25 & 45 & 32 & 33 & 20 \\
\hline 26 & 34 & 24 & 24 & 14 \\
\hline 27 & 502 & 122 & 39 & 38 \\
\hline 28 & 185 & 124 & 106 & 47 \\
\hline 29 & 26 & 6 & 24 & 4 \\
\hline 30 & 243 & 228 & 79 & 73 \\
\hline
\end{tabular}

Table 1: Homogeneous c.c.s and 2-closed groups 
Thus, the following implications hold:

Proposition 1.2 A symmetric c.c. is commutative; a commutative c.c. is stratifiable; and a stratifiable c.c. is homogeneous.

None of these implications reverses.

We note also that, if $\mathcal{P}=\mathcal{K}(G)$, then $\mathcal{P}$ is trivial if and only if $G$ is doubly transitive.

\subsection{History and terminology}

A symmetric coherent configuration is usually known as an association scheme. Association schemes were first used in the context of experimental design in statistics, by R. C. Bose and his school, as "carriers" of partially balanced designs [7]. The basis algebra of an association scheme, and the isomorphism to the intersection algebra, were constructed by Bose and Mesner [6], and for this reason the basis algebra is often called the Bose-Mesner algebra of the association scheme.

There are several reasons why only symmetric c.c.s are used. First, the relations which arise in practice in treatment and plot structures and the covariance matrices that arise are almost always symmetric. Indeed, these relations are often defined by concurrences in blocks. Secondly, statistical data consists of real numbers, and a large part of the work consists in computing orthogonal decompositions of real vector spaces; association schemes are a valuable tool for this purpose. In an association scheme, the basis matrices are commuting symmetric real matrices, and so the vector space $\mathbb{R}^{\Omega}$ has an orthogonal decomposition into common eigenspaces of these matrices. These eigenspaces are called strata. (This is the origin of the term "stratifiable".) Further, the Moore-Penrose inverse of any matrix in $\mathrm{BA}(\mathcal{P})$ is also in $\mathrm{BA}(\mathcal{P})$.

General coherent configurations were defined at about the same time by Higman [17] and by Weisfeiler and Leman [34]; the latter used the term cellular algebra for the algebra generated by the basis matrices. Subsequently, Delsarte in his thesis [9] showed the importance of association schemes in coding theory. Though his discussion applies to any commutative c.c. (and he extended the usage of the term "association scheme" to this class), his important examples are all symmetric. Bannai [5], in a recent survey, uses the term "non-commutative association scheme" for a homogeneous coherent configuration. More recently, there have been signs that the term "association scheme" or "scheme" is being applied to any coherent configuration (see [11], for example). 
I propose that this term should be restricted to its original meaning of "symmetric coherent configuration". This proposal is motivated in part by the large numbers of papers on association schemes in the statistical literature (see the references in [4]). I have adopted my proposal in this paper.

Symmetric matrices are so pervasive in statistics that, in the study of estimation of variance components, the fourth condition in the definition of a coherent configuration (closure under multiplication) is sacrificed; instead, closure under the Jordan product $A \circ B=\frac{1}{2}(A B+B A)$ is required. (This product is commutative and preserves symmetry of matrices.) Then Wedderburn's theorem on simple associative algebras must be replaced by some form of the Jordan-von NeumannWigner theorem on simple Jordan algebras. See Jacobson [23] for the theory of Jordan algebras, and Malley [27] for the statistical applications.

It is worth recording here a question to which I don't know the answer. Define a Jordan scheme to be a partition $\mathcal{P}$ of $\Omega^{2}$ with the properties that the diagonal is a single part, every part is symmetric, and there are numbers $q_{i j}^{k}$ such that

$$
A\left(R_{i}\right) A\left(R_{j}\right)+A\left(R_{j}\right) A\left(R_{i}\right)=\sum_{k=1}^{s} q_{i j}^{k} A\left(R_{k}\right) .
$$

The span of the symmetric matrices $A\left(R_{i}\right)$ over the real numbers is thus a Jordan algebra, and contains the Moore-Penrose inverse of each of its elements.

Clearly, the partition $Q^{\mathrm{sym}}$ obtained by symmetrising a homogeneous coherent configuration is a Jordan scheme. Are there any others?

The fourth column in Table 1 gives the number of association schemes on $n$ points for $n \leq 30$. This number is obtained from the information provided by Hanaki and Miyamoto [15], simply by checking which of the configurations are symmetric. (As noted earlier, in the case $n=29$, the classification of strongly regular graphs by Spence [33] is also used.) The fifth column lists the number of generously transitive groups of degree $n$ which are 2-closed, or equivalently the number of Schurian association schemes. The smallest number of points in a nonSchurian association scheme is 16; an example of such a scheme is the Shrikhande strongly regular graph [31].

Note also that the term "cellular algebra" has been used with an entirely different meaning by Graham and Lehrer [14] and in a number of subsequent papers. 


\section{The partial order}

The set of partitions of $\Omega \times \Omega$ forms a lattice, under the ordering given by $\mathcal{P} \preceq Q$ if $\mathcal{P}$ refines $Q$ (that is, every $\mathcal{P}$-class is contained in a $Q$-class). We use the symbol $\checkmark$ to denote the join in this lattice. Thus, $\left(\alpha_{1}, \alpha_{2}\right)$ and $\left(\beta_{1}, \beta_{2}\right)$ lie in the same $\mathcal{P} \vee Q$-class if it is possible to move from $\left(\alpha_{1}, \alpha_{2}\right)$ to $\left(\beta_{1}, \beta_{2}\right)$ by a sequence of moves each within either a $\mathcal{P}$-class or a $Q$-class.

The following result is due to Higman [19]; the short proof here is due to Bailey [3].

Theorem 2.1 The join (in the partition lattice) of two c.c.s is a c.c. The same holds for homogeneous, stratifiable, commutative, or symmetric c.c.s.

Proof A function is constant on the classes of $\mathcal{P} \vee Q$ if and only if it is constant on both the $\mathcal{P}$-classes and the $Q$-classes. So $\mathrm{BA}(\mathcal{P} \vee Q)=\operatorname{BA}(\mathcal{P}) \cap \mathrm{BA}(Q)$.

From this, we deduce the following result.

Theorem 2.2 The coherent configurations on $\Omega$ form a lattice.

Proof We must show that any two coherent configurations have a greatest lower bound. But the g.l.b. of $\mathcal{P}$ and $Q$ is the join of all the coherent configurations lying below both $\mathcal{P}$ and $Q$; and this set is non-empty, since it contains at least the discrete c.c. (Note that the meet of two coherent configurations is not the same as their meet as partitions!)

This argument fails for any of the other classes of coherent configurations, since there may be no c.c. in the appropriate class below any given $\mathcal{P}$ and $Q$. We will see examples for association schemes later.

Theorem 2.1 also has the following consequence.

Corollary 2.3 Let $\mathcal{P}$ be any partition of $\Omega^{2}$. Then there is a unique coherent configuration $\mathcal{P}^{*}$ on $\Omega$ which is maximal with respect to being a refinement of $\mathcal{P}$. Moreover, $\operatorname{Aut}(\mathcal{P})=\operatorname{Aut}\left(\mathcal{P}^{*}\right)$.

Proof The set of coherent configurations below $\mathcal{P}$ is non-empty (since it contains the discrete c.c.) and so has a supremum. The construction shows that any permutation preserving $\mathcal{P}$ will preserve $\mathcal{P}^{*}$. The converse holds since $\mathcal{P}^{*}$ refines $\mathcal{P}$. 
The coherent configuration $\mathcal{P}^{*}$ can be computed efficiently from $\mathcal{P}$. First, replacing $\mathcal{P}$ by $\mathcal{P} \wedge \mathcal{P}^{\top}$ if necessary (where $\mathcal{P}^{\top}$ is the partition whose parts are the converses of the parts of $\mathcal{P}$ ), we can assume that $\mathcal{P}=\mathcal{P}^{\top}$. Let $\mathcal{P}^{\prime}$ be the partition in which two ordered pairs $\left(\alpha_{1}, \beta_{1}\right)$ and $\left(\alpha_{2}, \beta_{2}\right)$ are in the same part whenever, for any two parts $X, Y$ of $\mathcal{P}$, the numbers of points $\gamma \in \Omega$ for which $\left(\alpha_{i}, \gamma\right) \in X$ and $\left(\gamma, \beta_{i}\right) \in Y$ are equal for $i=1,2$. Clearly, if $\mathcal{P}=\mathcal{P}^{\top}$, then $\mathcal{P}^{\prime}=\left(\mathcal{P}^{\prime}\right)^{\top}$. Now set $\mathcal{P}_{0}=\mathscr{P}$ and $\mathcal{P}_{n+1}=\mathcal{P}_{n}^{\prime}$ for $n \geq 0$. There exists $n$ such that $\mathcal{P}_{n}=\mathcal{P}_{n+1}$; set $\mathcal{P}^{*}=\mathcal{P}_{n}$. This is the required coherent configuration.

This provides a useful reduction for the problem of finding the automorphism group of a partition of $\Omega^{2}$ : we may assume that the partition is a coherent configuration. It also explains why coherent configurations provide difficult test cases for this problem, since this reduction gives no extra information in this case. This problem includes the problem of finding the automorphism group of a graph (consider the partition of $\Omega^{2}$ into the diagonal, the set of edges, and the set of nonedges of the graph). This is the original context in which coherent configurations arose in the work of Weisfeiler and Leman [34].

Coherent configurations were also used by the Soviet school for establishing the maximality of certain subgroups of symmetric and alternating groups, using the following principle:

Proposition 2.4 Let $G$ be a 2-closed permutation group on $\Omega$. Suppose that no coherent configuration lies strictly between $\mathcal{K}(G)$ and the trivial configuration on $\Omega$. Then any proper supergroup of $G$ in $\operatorname{Sym}(\Omega)$ is doubly transitive.

For example, Kaluznin and Klin [25] verified the maximality in this sense of $\mathcal{K}(G)$, where $G$ is the symmetric group of degree $n$ in its action on $m$-sets, for $n$ sufficiently large in terms of $m$, and thus showed the maximality of $G$ in the symmetric or alternating group of degree $\left(\begin{array}{l}n \\ m\end{array}\right)$.

Much more is known now about maximality, but the proofs typically use the classification and subgroup structure of the finite almost-simple groups (see [26]). As a result, many interesting questions on the maximality of $\mathcal{K}(G)$ for various groups $G$ remain unanswered! (See Faradžev et al. [12] for an account of some of the results that have been obtained.)

At about the same time, Kageyama [24] was considering the same question for association schemes, motivated by the question of when the number of associate classes in a partially balanced incomplete block design could be reduced.

We conclude this section with a curiosity. Let $\Lambda_{n}$ denote the lattice of c.c.s on a fixed $n$-set, say $\{0,1, \ldots, n-1\}$. 
Proposition 2.5 For any $n$, there is an embedding of $\Lambda_{n}$ as a down-set in $\Lambda_{n+1}$ preserving zero, join and meet.

Proof Let $\mathcal{P}$ be a c.c. on $\{0,1, \ldots, n-1\}$, with fibres $F_{1}, \ldots, F_{t}$. We define $F(\mathcal{P})$ to be the partition of $\{0, \ldots, n\}^{2}$ consisting of the following sets of pairs: all the parts of $\mathcal{P}$; the sets $\left\{(x, n): x \in F_{i}\right\}$ and their converses for $i=1, \ldots, t$; and the set $\{(n, n)\}$. It is not difficult to show that $F(\mathcal{P})$ is a c.c., and that every c.c. that has $\{(n, n)\}$ as one of its parts arises in this way. Moreover, $F$ maps the discrete c.c. to the discrete c.c. and preserves join and meet.

Note that the direct limit of these lattices and maps is the "lattice of all finite coherent configurations". (The direct limit contains no top element; this has to be adjoined.)

\section{AS-friendly and AS-free groups}

In the remainder of this paper, we will be concerned with association schemes. Although there is a coherent configuration $\mathcal{K}(G)$ associated with every permutation group $G$ (and this configuration is trivial if and only if the group is doubly transitive), this is far from being the case for association schemes. Since any association scheme is a homogeneous coherent configuration, I will consider only transitive permutation groups in this section, which reports on work of Alejandro et al. [1]. I have sketched some of the proofs, but refer to [1] for full details.

We begin with some definitions. Let $G$ be a transitive permutation group on the finite set $\Omega$.

(a) We say that $G$ is $A S$-free if the only $G$-invariant association scheme on $\Omega$ is the trivial scheme.

(b) We say that $G$ is $A S$-friendly if there is a unique minimal $G$-invariant association scheme on $\Omega$.

Of course, if we replaced "AS" by "CC" in the above definitions, then every group would be CC-friendly, and the CC-free groups would be precisely the doubly transitive groups.

Note that a 2-homogeneous group $G$ (one which is transitive on the 2-element subsets of $\Omega$ ) is AS-free, since the symmetrisation of $\mathcal{K}(G)$ is the trivial configuration. 
It is difficult, both theoretically and computationally, to decide whether a transitive group $G$ of large rank is AS-friendly or AS-free: we have to merge the symmetrised orbitals in all possible ways and check whether an association scheme is obtained. The following idea can be used to reduce the search for $G$-invariant association schemes. Suppose that the permutation character of $G$ has the form $\pi=\sum e_{i} \chi_{i}$, where the $\chi_{i}$ are distinct irreducible characters. Then the rank of $G$ (the number of orbits on $\Omega^{2}$ ) is equal to $\sum e_{i}^{2}$, while the dimension of the largest commutative semisimple subalgebra of $\operatorname{BA}(\mathcal{K}(G))$ is $\sum e_{i}$. So, if some of the multiplicities $e_{i}$ are large, then a lot of merging is required.

\subsection{Basic theory}

We begin with an example of a group which is not AS-friendly. Let $G$ be the symmetric group $S_{n}$ (for $n \geq 5$ ), acting on the set $\Omega$ of ordered pairs of distinct elements from the set $\{1, \ldots, n\}$ : we write the pair $(i, j)$ as $i j$ for brevity. The coherent configuration $\mathcal{K}(G)$ consists of the following parts:

$$
\begin{aligned}
& R_{1}=\{(i j, i j): i \neq j\}, \\
& R_{2}=\{(i j, j i): i \neq j\}, \\
& R_{3}=\{(i j, i k): i, j, k \text { distinct }\}, \\
& R_{4}=\{(i j, k j): i, j, k \text { distinct }\}, \\
& R_{5}=\{(i j, k i): i, j, k \text { distinct }\}, \\
& R_{6}=\{(i j, j k): i, j, k \text { distinct }\}, \\
& R_{7}=\{(i j, k l): i, j, k, l \text { distinct }\} .
\end{aligned}
$$

We have $R_{5}^{\top}=R_{6}$; all other relations are symmetric. The symmetrised partition is not an association scheme, but we find three minimal association schemes as follows:

- the pair scheme: $\left\{R_{1}, R_{2}, R_{3} \cup R_{4}, R_{5} \cup R_{6}, R_{7}\right\}$ (see [24, pp. 576-578]);

- two "divisible" schemes $\left\{R_{1}, R_{3}, R_{2} \cup R_{4} \cup R_{5} \cup R_{6} \cup R_{7}\right\}$ and $\left\{R_{1}, R_{4}, R_{2} \cup\right.$ $\left.R_{3} \cup R_{5} \cup R_{6} \cup R_{7}\right\}$.

These are all incomparable, so there is not a unique minimal association scheme.

The next result shows how the concepts just defined are related to more familiar concepts of permutation group theory. 
Theorem 3.1 The following implications hold between properties of a permutation group $G$ :

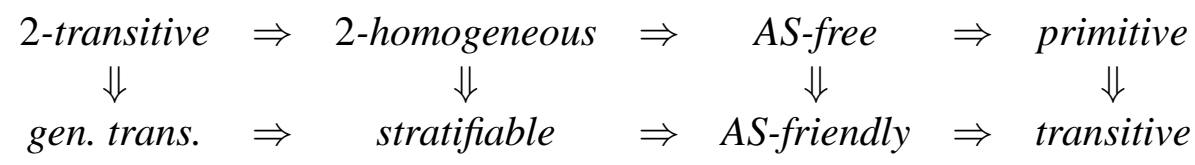

None of these implications reverses, and no further implications hold.

Since the properties of a permutation group $G$ listed in the theorem depend only on $\mathcal{K}(G)$, it suffices to consider 2 -closed groups. Table 2 gives the numbers of 2-closed groups of small degree which are respectively 2-transitive, 2homogeneous, AS-free, primitive, generously transitive, stratifiable, AS-friendly, and transitive. Note that up to degree 30, every AS-free group is 2-homogeneous and every AS-friendly group is stratifiable.

The smallest 2-closed primitive group which is not $\operatorname{AS}$-friendly is $\operatorname{PSL}(2,11)$, with degree 55. The smallest 2-closed primitive groups which are AS-friendly but not stratifiable are $\operatorname{PSL}(2,13)$, with degrees 78 and 91 . These groups are numbers $(55,1),(78,1),(91,1)$ and $(91,3)$ in the list of primitive groups available in GAP. The smallest examples of AS-free groups which are not stratifiable have degree 234, and are isomorphic to $\operatorname{PSL}(3,3)$ and $\operatorname{PSL}(3,3): 2$, numbers $(234,1)$ and $(234,2)$ in the list. (Further examples of such groups will be given later.) 2-homogeneous groups which are not generously transitive are well known (such groups must have prime power degree congruent to 3 mod 4). I hope to make the GAP code used for these tests available shortly.

The class of AS-friendly groups is also closed under taking supergroups, wreath products, and primitive components:

Theorem 3.2 (a) If a group has an AS-friendly subgroup, then it is AS-friendly.

(b) The class of AS-friendly permutation groups is closed under wreath product (with the imprimitive action).

(c) Let $G$ be imprimitive; let $\Gamma$ be a system of imprimitivity and $\Delta$ a block in $\Gamma$, and let $H$ be the permutation group induced on $\Delta$ by its setwise stabiliser and $K$ the group induced on $\Gamma$ by $G$, so that $G \leq H$ 乙 $K$. If $G$ is $A S$-friendly, then so are $H$ and $K$.

(d) The same assertions hold with "stratifiable" or "generously transitive" in place of "AS-friendly". 


\begin{tabular}{|r||r|r|r|r||r|r|r|r||}
\hline$n$ & $2 \mathrm{~T}$ & $2 \mathrm{H}$ & $\mathrm{A} \Phi$ & $\mathrm{Pr}$ & $\mathrm{GT}$ & $\mathrm{St}$ & $\mathrm{AF}$ & $\mathrm{Tr}$ \\
\hline 1 & 1 & 1 & 1 & 1 & 1 & 1 & 1 & 1 \\
2 & 1 & 1 & 1 & 1 & 1 & 1 & 1 & 1 \\
3 & 1 & 2 & 2 & 2 & 1 & 2 & 2 & 2 \\
4 & 1 & 1 & 1 & 1 & 3 & 4 & 4 & 4 \\
5 & 1 & 1 & 1 & 3 & 2 & 3 & 3 & 3 \\
6 & 1 & 1 & 1 & 1 & 4 & 7 & 7 & 8 \\
7 & 1 & 2 & 2 & 4 & 2 & 4 & 4 & 4 \\
8 & 1 & 1 & 1 & 1 & 10 & 20 & 20 & 21 \\
9 & 1 & 1 & 1 & 2 & 6 & 12 & 12 & 12 \\
10 & 1 & 1 & 1 & 2 & 8 & 11 & 11 & 13 \\
11 & 1 & 2 & 2 & 4 & 2 & 4 & 4 & 4 \\
12 & 1 & 1 & 1 & 1 & 21 & 47 & 47 & 59 \\
13 & 1 & 1 & 1 & 6 & 4 & 6 & 6 & 6 \\
14 & 1 & 1 & 1 & 1 & 8 & 14 & 14 & 16 \\
15 & 1 & 1 & 1 & 2 & 10 & 23 & 23 & 24 \\
16 & 1 & 1 & 1 & 4 & 56 & 171 & 171 & 206 \\
17 & 1 & 1 & 1 & 5 & 4 & 5 & 5 & 5 \\
18 & 1 & 1 & 1 & 1 & 32 & 71 & 71 & 93 \\
19 & 1 & 2 & 2 & 6 & 3 & 6 & 6 & 6 \\
20 & 1 & 1 & 1 & 1 & 41 & 73 & 73 & 95 \\
21 & 1 & 1 & 1 & 3 & 11 & 29 & 29 & 32 \\
22 & 1 & 1 & 1 & 1 & 8 & 14 & 14 & 16 \\
23 & 1 & 2 & 2 & 4 & 2 & 4 & 4 & 4 \\
24 & 1 & 1 & 1 & 1 & 136 & 454 & 454 & 669 \\
25 & 1 & 1 & 1 & 9 & 20 & 32 & 32 & 32 \\
26 & 1 & 1 & 1 & 1 & 14 & 20 & 20 & 24 \\
27 & 1 & 2 & 2 & 5 & 38 & 112 & 112 & 122 \\
28 & 1 & 1 & 1 & 4 & 47 & 103 & 103 & 124 \\
29 & 1 & 1 & 1 & 6 & 4 & 6 & 6 & 6 \\
30 & 1 & 1 & 1 & 1 & 73 & 166 & 166 & 228 \\
\hline
\end{tabular}

Table 2: Small 2-closed permutation groups 


\subsection{Regular groups}

Groups whose regular representation is AS-friendly have been determined. In particular, the properties "AS-friendly" and "stratifiable" coincide for regular groups.

Following Bailey [3], a partition $P=\left\{P_{1}, \ldots, P_{s}\right\}$ of a group $G$ is called a blueprint if the partition $\mathcal{P}=\left\{R_{1}, \ldots, R_{s}\right\}$ of $G \times G$ given by

$$
R_{i}=\left\{(x, y): x y^{-1} \in P_{i}\right\}
$$

is a coherent configuration on $G$. Note that this coherent configuration is invariant under right translation by $G$. This condition is equivalent to the assertion that the sums (in the group ring $\mathbb{Z} G$ ) of the classes $P_{1}, \ldots, P_{S}$ span a Schur ring: see Wielandt [35].

The inverse partition of a group $G$ is the partition whose parts are the sets $\left\{g, g^{-1}\right\}$ for $g \in G$.

Theorem 3.3 For a finite group $G$, the following four conditions are equivalent:

(a) the regular action of $G$ is $A S$-friendly;

(b) the regular action of $G$ is stratifiable;

(c) the inverse partition of $G$ is a blueprint;

(d) either $G$ is abelian, or $G \cong Q \times A$ where $Q$ is the quaternion group of order 8 and $A$ is an elementary abelian 2-group.

Proof I will outline briefly the two non-trivial parts of the proof.

Clearly (c) implies (b) implies (a). To show that (a) implies (c), we proceed as follows. Let $P$ be the minimal blueprint on $G$; we must show that $P$ is the inverse partition. So take an element $g \in G$; we must show that $(1, h)$ lies in the same class as $(1, g)$ if and only if $h=g^{ \pm 1}$. Now the right cosets of $H=\langle g\rangle$ form a system of imprimitivity, and so $G \leq H<S_{m}$, where $m=|G: H|$. This group preserves the association scheme obtained by "nesting" the cyclic scheme on $H$ in the trivial scheme on $m$ points, so the unique minimal $G$-invariant scheme is contained in this one. But in this scheme, we see that the result holds.

Also, the proof that (d) implies (b) uses relatively straightforward representation theory. Suppose that a group satisfies (c). It is easy to see that, given any two elements $g, h \in G$, either $g$ and $h$ commute, or each inverts the other. Thus every subgroup of $G$ is normal, and the structure is determined by the theorem of Dedekind (see [22], Satz 7.12 on p. 308) and a little more work. 


\subsection{Primitive groups}

We have seen that 2-homogeneous groups are AS-free. Are there any other transitive AS-free groups?

A permutation group is called non-basic if there is a bijection between $\Omega$ and $\Gamma^{\Delta}$ (the set of functions from $\Delta$ to $\Gamma$ ) for some finite sets $\Gamma$ and $\Delta$, which induces an isomorphism from $G$ to a subgroup of $\operatorname{Sym}(\Gamma)$ \& $\operatorname{Sym}(\Delta)$ with the product action. This concept arises in the O'Nan-Scott classification of primitive permutation groups, see [10], p. 106. Of course, a group is basic if it is not non-basic.

Theorem 3.4 Let $G$ be a transitive AS-free group. Then $G$ is primitive and basic, and is 2-homogeneous, diagonal or almost simple.

Proof An imprimitive permutation group $G$ preserves the "divisible" association scheme whose parts are the diagonal, the $G$-congruence with the diagonal removed, and the rest of $\Omega \times \Omega$, while a non-basic group preserves a Hamming scheme (see Delsarte [9]). By the O'Nan-Scott theorem, basic primitive groups are affine, diagonal, or almost simple. An affine group has an abelian regular normal subgroup and thus is stratifiable; so if such a group is AS-free, then it is 2-homogeneous.

Almost simple AS-free groups which are not 2-homogeneous do exist. This can be seen from the paper of Faradžev et al. [12]. These authors consider the following problem. Let $G$ be a simple primitive permutation group of order at most $10^{6}$ but not $\operatorname{PSL}(2, q)$. Describe the coherent configurations above $\mathcal{K}(G)$. Table 3.5.1 on p. 115 gives their results. In several cases, no non-trivial configuration consists entirely of symmetric matrices: such groups are of course AS-free. The smallest example is the group $\operatorname{PSL}(3,3)$, acting on the right cosets of $\operatorname{PO}(3,3)$ (a subgroup isomorphic to $S_{4}$ ), with degree 234; as we have seen, this is the smallest AS-free group which is not 2-homogeneous. Other examples of AS-free groups in this list are $M_{12}$, degree $1320 ; J_{1}$, degree 1463,1540 or 1596 ; and $J_{2}$, degree 1800. The situation is not well understood!

The table also gives another example of a primitive group which is not ASfriendly; this is $M_{12}$, with degree 495.

No AS-free primitive diagonal group is known at present. It is known that the socle of such a group must have at least four simple factors: 
- A primitive diagonal group whose socle has two factors is a group of weak automorphisms of the conjugacy class configuration of a simple group (corresponding to the blueprint formed by the conjugacy classes), and indeed $\mathcal{K}(G)$ is commutative for such groups $G$ (so they are stratifiable).

- A primitive diagonal group whose socle has three factors preserves a Latin square scheme based on the Cayley table of the simple group. However, it is not known whether such groups are AS-friendly.

Thus, the smallest possible degree of an AS-free diagonal group is 216000.

In the next subsection I report on a more general investigation of diagonal groups (not necessarily primitive).

\subsection{Diagonal groups}

The diagonal group $D(T, n)$, where $T$ is a group and $n$ a positive integer, is defined as the permutation group on the set

$$
\Omega=T^{n}=\left\{\left[x_{1}, \ldots, x_{n}\right]: x_{1}, \ldots, x_{n} \in T\right\}
$$

generated by the following permutations:

(a) the group $T^{n}$ acting by right translation, that is, the permutations

$$
\left[x_{1}, \ldots, x_{n}\right] \mapsto\left[x_{1} t_{1}, \ldots, x_{n} t_{n}\right]
$$

$$
\text { for } t_{1}, \ldots, t_{n} \in T \text {; }
$$

(b) the automorphism group of $T$, acting coordinatewise, that is,

$$
\left[x_{1}, \ldots, x_{n}\right] \mapsto\left[x_{1}^{\alpha}, \ldots, x_{n}^{\alpha}\right]
$$

for $\alpha \in \operatorname{Aut}(T)$;

(c) the symmetric group $S_{n}$, acting by permuting the coordinates, that is,

$$
\pi:\left[x_{1}, x_{2}, \ldots, x_{n}\right] \mapsto\left[x_{1 \pi}, x_{2 \pi}, \ldots, x_{n \pi}\right]
$$

for $\pi \in S_{n}$;

(d) the permutation

$$
\tau:\left[x_{1}, x_{2}, \ldots, x_{n}\right] \mapsto\left[x_{1}^{-1}, x_{1}^{-1} x_{2}, \ldots, x_{1}^{-1} x_{n}\right] .
$$


The group $D(T, n)$ is a "maximal diagonal group"; by Theorem 3.2, if any diagonal group having a normal subgroup $T^{n+1}$ acting on the cosets of the diagonal is generously transitive, stratifiable, or AS-friendly, then $D(T, n)$ will have this property. If $T$ is abelian, then $D(T, n)$ has an abelian regular normal subgroup consisting of the permutations of type (a), and so is AS-friendly (and even stratifiable). For non-abelian groups $T$, it is not known when $D(T, n)$ can be ASfriendly. However, the following result is known about generous transitivity and stratifiability.

Theorem 3.5 Let $T$ be a non-abelian finite group.

(a) If $D(T, n)$ is stratifiable, then $n \leq 8$; and if $D(T, n)$ is generously transitive, then $n \leq 7$.

(b) The group $D(T, 7)$ is generously transitive if and only if $T$ is the quaternion group of order 8 .

Proof The group $D(T, n)$ is generously transitive if and only if every $n$-tuple of elements of $T$ can be inverted by a combination of transformations of types (b)(d). If $T$ is non-abelian, it is shown that this is impossible for suitably chosen 8tuples, while for 7-tuples we find that any two non-commuting elements generate $Q_{8}$. The argument for stratifiability is similar but a bit more complicated.

The group $D(T, n)$ is primitive if and only if $T$ is characteristically simple, and is AS-free only if $T$ is simple. It seems likely that the bounds in the theorem can be improved for non-abelian simple groups $T$.

Acknowledgment I am grateful to P. P. Alejandro, R. A. Bailey, A. Hanaki, A. Hulpke, M. H. Klin, L. H. Soicher and E. Spence for helpful comments.

\section{References}

[1] P. P. Alejandro, R. A. Bailey and P. J. Cameron, Association schemes and permutation groups, Discrete Math., submitted.

[2] R. A. Bailey, Strata for randomized experiments, J. Royal Statist. Soc. (B) 53 (1991), 27-78. 
[3] R. A. Bailey, Suprema and infima of association schemes, Discrete Math., to appear.

[4] R. A. Bailey, Association Schemes: Designed Experiments, Algebra and Combinatorics, in preparation.

[5] E. Bannai, An introduction to association schemes, Methods of Discrete Mathematics (S. Löwe, F. Mazzocca, N. Melone and U. Ott, eds.), Quaderni di Mathematica 5, Seconda Università di Napoli, Napoli, 1999, pp. 1-70.

[6] R. C. Bose and D. M. Mesner, On linear associative algebras corresponding to association schemes of partially balanced designs, Ann. Math. Statist. 30 (1959), 21-38.

[7] R. C. Bose and K. R. Nair, Partially balanced incomplete block designs, Sankhyä 4 (1939), 337-372.

[8] J. H. Conway, A. Hulpke and J. McKay, On transitive permutation groups, London Math. Soc. J. Comput. Math. 1 (1998), 1-8,

http://www. lms.ac.uk

[9] Ph. Delsarte, An algebraic approach to the association schemes of coding theory, Philips Research Reports Suppl. 10 (1973).

[10] J. D. Dixon and B. Mortimer, Permutation Groups, Graduate Texts in Mathematics 163, Springer-Verlag, New York, 1996.

[11] S. Evdokimov and I. Ponomarenko, Separability number and Schurity number of coherent configurations, Electronic J. Combinatorics 7(1) (2000), \#R31, http: / / www. combinatorics.org

[12] I. A. Faradžev, M. H. Klin and M. E. Muzichuk, Cellular rings and groups of automorphisms of graphs, in Investigations in Algebraic Theory of Combinatorial Objects (I. A. Faradžev, A. A. Ivanov, M. H. Klin and A. J. Woldar, eds.), Kluwer, Dordrecht, 1994, pp. 1-152.

[13] The GAP Group, GAP — Groups, Algorithms, and Programming, Version 4.2; Aachen, St Andrews, 1999,

http://www-gap.dcs.st-and.ac.uk/ gap

[14] J. J. Graham and G. I. Lehrer, Cellular algebras, Invent. Math, 123 (1996), $1-34$. 
[15] A. Hanaki and I. Miyamoto, Classification of association schemes with small vertices, http://kissme.shinshu-u.ac.jp/as/

[16] D. G. Higman, Finite permutation groups of rank 3, Math. Z. 86 (1964), $145-156$.

[17] D. G. Higman, Intersection matrices for finite permutation groups, J. Algebra 6 (1967), 22-42.

[18] D. G. Higman, Combinatorial Considerations about Permutation Groups, Mathematical Institute, Oxford, 1971.

[19] D. G. Higman, Coherent algebras, Linear Algebra Appl. 93 (1987), 209-239.

[20] A. Hulpke, Constructing transitive permutation groups, in preparation.

[21] A. Hulpke, Transitive groups of small degree, http://www.math.ohio-state.edu/ ahulpke/smalldeg.html

[22] B. Huppert, Endliche Gruppen I, Grundlehren der mathematischen Wissenschaften 134, Springer-Verlag, Berlin, 1967.

[23] N. Jacobson, Structure and Representation of Jordan Algebras, American Mathematical Society, Providence, RI, 1968.

[24] S. Kageyama, Reduction of associate classes for block designs and related combinatorial arrangements, Hiroshima Math. J. 4 (1974), 527-618.

[25] L. A. Kaluznin and M. H. Klin, On certain maximal subgroups of symmetric and alternating groups, Math. USSR Sbornik 16 (1972), 95-123.

[26] M. W. Liebeck, C. E. Praeger and J. Saxl, The classification of the maximal subgroups of the finite symmetric and alternating groups, J. Algebra 111 (1987), 365-383.

[27] J. D. Malley, Optimal Unbiased Estimation of Variance Components, Lecture Notes in Statistics 39, Springer-Verlag, Berlin, 1986.

[28] B. D. McKay, nauty user's guide (version 1.5), Technical report TR-CS90-02, Computer Science Department, Australian National University, 1990.

[29] B. D. McKay and E. Spence, in preparation; see http://gauss.maths.gla.ac.uk/ ted/srgraphs.html 
[30] L. Pyber, Asymptotic results for permutation groups, Groups and Computation (L. Finkelstein and W M. Kantor, eds.), DIMACS Series in Discrete Mathematics and Theoretical Computer Science 11, American Mathematical Society, Providence, RI, 1993, pp. 197-219.

[31] S. S. Shrikhande, The uniqueness of the $L_{2}$ association scheme, Ann. Math. Statistics 30 (1959), 781-798.

[32] L. H. Soicher, GRAPE: A system for computing with graphs and groups, in Groups and Computation (L. Finkelstein and W. M. Kantor, eds.), DIMACS Series in Discrete Mathematics and Theoretical Computer Science 11, American Mathematical Society, Providence, RI, 1993, pp. 287-291.

[33] E. Spence, Strongly regular graphs on at most 64 vertices, http://gauss.maths.gla.ac.uk/ ted/srgraphs.html

[34] B. Yu. Weisfeiler and A. A. Leman, Reduction of a graph to a canonical form and an algebra which appears in the process, Scientific-Technological Investigations (2) 9 (1968), 12-16.

[35] H. Wielandt, Finite Permutation Groups, Academic Press, New York, 1964.

[36] P.-H. Zieschang, Homogeneous coherent configurations as generalized groups and their relationship to buildings, J. Algebra 178 (1995), 677-709. 